\title{
How should we communicate, listen and respond to patient in ophthalmology?
}

Keywords: health care system, community, eye contact, patients, disease, clinicians

\section{Commentary}

In this current age, the importance of the patient's role in improving our healthcare system and the level of care we provide is more than evident. As a healthcare professional, looking at one's practice from the patient's aspect allows you to notice, amend and develop many aspects which you will never be able to do alone. Additionally, unlike the old days, when engaging with patients and obtaining their opinion was difficult, often leaving the doctor isolated in his clinic and away from his community, things have changed and more flexible interaction methods are now present. Having an eye disease could be life changing; therefore, we should ensure that we listen properly to what patients feel and say. This should take place on both an individual and organisational basis; for example, when being involved in a discussion with a patient, one need to maintain eye contact, allow sufficient time for the person to express their views and feelings, be reassuring by showing empathy and support and ensure all concerns are taken seriously. These are critical points when listening to what patients say.

In addition, healthcare organisations should set up clear, flexible and structured channels to receive feedback, concerns and thoughts from their patients. Furthermore, special teams might be allocated for this mission, thus building bridges of trust between patients and their doctors. Additionally, contacting everyone who has used the service recently, as well as offering more accessible ways of communication like online forms, an email service and even via a phone call, are all helpful in ensuring patients have a voice. Moreover, what makes this whole process valuable is when the information received is analysed, not over looked before reaching its destination and without achieving its purpose and goal. For instance, setting up a feedback service with no one monitoring it and taking appropriate actions is a waste of time, resources and effort for everyone. It also needs to be impartially managed; objectivity is essential for its success.

Moving forward, one of the actions that can be taken to respond to
Volume 10 Issue I - 2020

\author{
Housam Monla Haidar \\ Cardiff and Vale University Health Board, UK \\ Correspondence: Housam Monla Haidar, Cardiff and Vale \\ University Health Board, Cardiff, UK, \\ Email Housam.haidar@doctors.org.uk \\ Received: January 02, 2020 | Published: February 06, 2020
}

the patient's voice is to organise meetings between relevant teams and on different levels to discuss, analyse and react appropriately in a way that meets the patient's expectations and needs. It is also undeniable how important it is to engage the patients themselves in any plan or solution as this would avoid acting inappositely, reassuring them that effort is being made to improve the service based on the information that they have provided. In the following phase, feedback on the action that has been taken should be obtained, helping managers to ensure that their service listens to patients and maintains the highest possible standards, thus taking the relationship between the patient and his clinician to a level of trust, leading to mutual benefits for both sides. Finally, contributing towards a sufficient response to what patients say is for everyone, from policy maker to healthcare professional, the ability to reflect on everything we hear, analyse and react to as this reflection would then be a positive method which helps to develop our skills and review our effectiveness.

\section{Conclusion}

To conclude, the health care system in the UK clearly aims to achieve the highest levels of care. To achieve this, an open and onset communication must be established between the patients and healthcare professionals; what patients say must be listened to, analysed, responded to and reflected on by researchers, clinicians, funders and policymakers, ensuring that all is aligned to the needs and priorities of those who experience disease. 